\title{
Whiskers, Hyperhemispheres, and a Honeymoon
}

\author{
David Rutledge, Division of Engineering and Applied Science, California Institute of Technol0gy, \\ Pasadena, California, USA
}

I plan to make a few historical comments on early research in antennas for submillimeter waves, as the area was known in the 1970's. The initial focus was on understanding the limits of the tungsten whiskers that were then used as antennas. However, as integrated-circuit technology improved, it became clear that planar antennas on semiconductor substrates were needed. However, these antennas interact strongly with substrate modes. One way to eliminate the substrate modes is to put a lens on the back of the substrate and to couple power in through the lens. These substrate-lens coupled antennas are widely used today in laboratory instruments and radio astronomy. Another approach to suppressing the substrate modes is through a periodic antenna structure. This is the basis for the grid amplifier, which combines the outputs of large numbers of transistors in free space. This allows much more transmitter power than was previously possible from solid-state devices. The Wavestream Corporation was founded to develop this technology. The company's bestselling product is a $50-\mathrm{W}$ amplifier for $30-\mathrm{GHz}$ satellite uplinks. Several thousand amplifiers have been sold to date. 